\title{
Handwritten Slides on a TabletPC in a Discrete Mathematics Course
}

\author{
Evan Golub \\ Department of Computer Science \\ Human-Computer Interaction Lab \\ University of Maryland \\ College Park, MD 20742 \\ egolub@acm.org
}

\begin{abstract}
There are a wide variety of ways to present information at the front of a classroom. These include chalk on a blackboard, markers on a whiteboard, pens on transparencies, and computer projection systems. While computer-based presentation systems provide many opportunities both in and out of the classroom, there may also be many limitations. In-class spontaneity and dynamic exposition might be restricted. Class preparation time might increase dramatically when compared to the amount required to prepare handwritten materials. Certain presentation techniques may no longer be available. This paper will introduce a computer-based presentation system modeled on handwritten transparencies. It will then discuss how it addresses the above issues as well as how it can be used in and out of the classroom. These will be explored in the context of its use while teaching an undergraduate discrete mathematics course.
\end{abstract}

\section{Categories and Subject Descriptors}

K.3 [Computers and Education]: Computer uses in Education Computer-assisted instruction (CAI).

General Terms: Design, Human Factors.

Keywords: Classroom presentations, discrete mathematics.

\section{INTRODUCTION}

Computers can be a powerful presentation resource in the classroom. Computers have been available in some classrooms since (at least) the 1970s [4,7]. Their first uses were often to show programs being written and executed. In the past decade, presentations using general-purpose tools such as PowerPoint and HTML documents, projects extending existing presentation tools such as PowerPoint $[2,5,6,10]$ or custom-built tools $[1,3,9,11]$ have become popular not only in programming classes, but across the curriculum and the campus at large.

Advantages of computer-assisted instruction (CAI) include a faster pace when desired, the ability to better prepare orderly

Permission to make digital or hard copies of all or part of this work for personal or classroom use is granted without fee provided that copies are not made or distributed for profit or commercial advantage and that copies bear this notice and the full citation on the first page. To copy otherwise, or republish, to post on servers or to redistribute to lists, requires prior specific permission and/or a fee.

SIGCSE'04, March 3-7, 2004, Norfolk, Virginia, USA

Copyright 2004 ACM 1-58113-798-2/04/0003 ...\$5.00. presentations, and ease of archiving and reuse of notes. However, there are many faculty members that have access to technology classrooms and still choose not to make use of that technology. Reasons for this range from the practical to the pedagogical to the philosophical.

This paper will only briefly address reasons why CAI is not being used in certain situations where practicality and pedagogy are at issue. It will then discuss which of these appear to have solutions potentially within reach. The remainder of the paper will discuss a presentation system developed by the author and how it was used successfully in a sophomore-level discrete mathematics course at the University of Maryland. This discussion will cover aspects of the preparation of classroom presentation material as well as discuss working with students, teaching assistants, and other faculty members.

\section{WHY NON-TECHNOLOGY PRESENTATIONS?}

Why do some people still use chalk, whiteboards, Mylar slides? There are two different general, though non-exclusive, categories into which reasons appear to fall based upon my observations and discussions with colleagues that I will discuss; time investment and flexibility.

Depending upon the course, preparing PowerPoint slides or HTML documents can be a very time consuming process. One needs to start concerning oneself with layout, font size, and color schemes. In a mathematical course, or on a mathematical topic, one needs to navigate equation editors. If a faculty member already has outlines prepared, or is able to present from memory, and typically writes notes on the board at the front of the room while they talk, their class preparation time could be minimal. If they need to convert those into pre-made slides, there is a potentially large and (hopefully) one-time cost. I say "hopefully" due to the fact that faculty who created decks of transparencies 15 years ago quite likely saw that as a one-time cost, but if they have found (or do find) value in a new system, there is a new time investment.

There is also the question of flexibility once the slides are created. If you notice an error on your slide, or think of something on the fly, stopping to edit your PowerPoint slide or going into your editor to modify and then reload your HTML document is more likely to break the flow of the lecture. You can either draw on top of a PowerPoint slide with your mouse or with a stylus on a TabletPC or other pen-aware computer. However, your space is 
limited, and it is useful if you can leave space in advance for where expect to add notes. This ability with PowerPoint is extended with Presenter [5], an application that has been in development at the University of Washington. In an HTML document, you could dynamically add material by inserting a text box into which you could type during class, though this still limits the placement of the additional notes. In either case, to a certain degree, you are now in a position where you must plan your spontaneity.

\section{THE TABLET MYLAR SLIDES APPLICATION}

Traditional Mylar slides (transparencies) fall into two categories; decks and rolls. With a slide deck, you have a set of individual slides, each slide being approximately the size of a sheet of paper. During class, you progress through the slides, using spare blanks as needed. Having individual slides supports the reorganization of material as well as insertion of new material. However, for a long concept, it could require you to physically divide the material across multiple slides. When doing this, you may need to consider where to make the division as well as how to convey to the audience that the change of slides does not necessarily indicate a change of concept or even a new stage of the concept.

With a roll, you have a roll of Mylar that is approximately the width of a piece of paper, but is the length of many sheets. Rolls are typically used with a projector that has a set of reels to allow you to scroll through the single long slide. However, this format does not encourage modification. In order to add, move, or remove material, you would need to cut the roll into parts, splicing in new material or splicing joins around moved or deleted material.

In either the individual-sheet or the long-roll scenario, the use of multiple pen colors to indicate stages, comments, or relative importance of information can be a valuable resource.

The Tablet Mylar Slides (TMS) application merges the best aspects of both of these formats into a single tool. You are able to have slides of whatever length you require, but still have the ability to add and delete material at will. It adds several features that are highly beneficial when working with a TabletPC as your presentation tool as well as providing you with benefits associated with storing your slides electronically.

The primary design goal for authoring in TMS was to have an interaction model that was as similar as possible to the feel of traditional transparencies. This included supporting the rapid creation of class notes, while still taking advantage of the fact that the environment was a computer. As an example, if after creating a slide you noticed that you omitted an item, you could simply select the space insertion tool and electronically push material lower on the page as you could in other electronic document creation applications. You could then add material without needing to erase and recreate what was below as you would on a traditional transparency.

For the presentation side, the primary goals were to be able to work either in portrait or landscape mode on the TabletPC (a nontrivial point), and to maintain the in-class flexibility of traditional transparencies. Though working in portrait mode actually reduces the number of pixels being utilized on the projector, it can still be desirable.

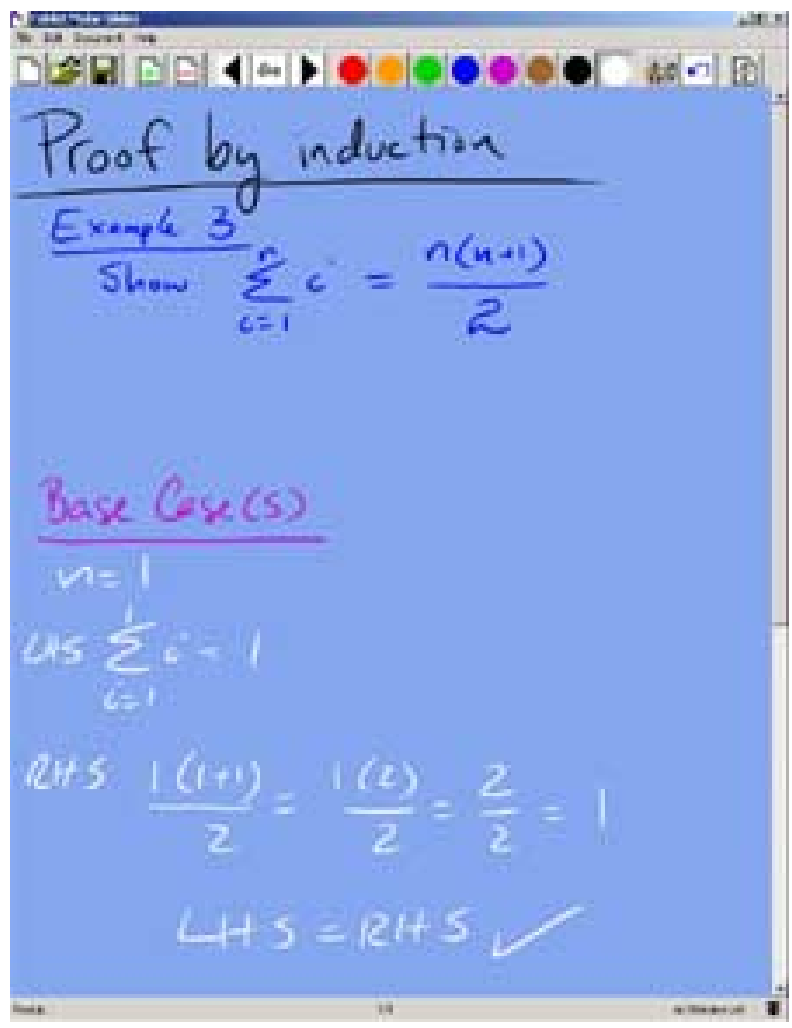

Figure 1: TMS Application faculty view with example notes for proof displayed.

In preparing transparencies for class, having long sheets is preferred by some to having wide sheets. If you choose to present notes in a visually linear form, you might tend to waste transparencies since you are not making use of the right-hand side of them. Additionally, it would require more breaks in the material. When preparing to transition to an electronic version using a TabletPC, I considered the issue that the ceiling mounted projector did not rotate 90 degrees. This meant that while in portrait mode on the computer, a portion of the projector's display would go unused. This is really no different from utilizing only part of a traditional overhead projector's platform.

There is, however, a potential benefit to working in portrait mode. When writing on my TabletPC it appeared that the size at which I wrote was no different when working in landscape or portrait mode. This meant that when working in portrait mode, I would still have more effective space on the screen at a given moment, just as the case had been when I previously opted to use transparencies oriented to be long rather than wide. The way I use my working space means that working in landscape mode would utilize more pixels, yet present less information.

The interface of the current version is modeled on selfobservations of the creation of traditional slides. The toolbar provides direct access to several file options, the ability to add or remove pages, navigation, a set of seven pens with user-definable colors for standard inking as well as one pen for "hidden" ink, 
preferences, one level of undo/redo and the space insertion tool. Palettes and additional toolbar items may be added to later versions; the choice and design of these will be informed by use of the current version.

When inking slides, the toolbar buttons for selecting pen colors provided the ability to quickly switch pen colors. If your stylus has a barrel button, you can depress it and then select ink with a lasso. Once selected, it can be moved, copied or cut. You can also paste ink selected from other slides or from other ink-enabled applications onto the current slide.

There is an ability to write in a "hidden" ink color. Material written with this ink is visible on the presentation machine, but is not visible on the projection screen. This supports the ability to write notes to yourself as you might on the white backing sheet for a transparency. Figure 1 shows a sample class slide in which the example problem is shown, the outline for the proof technique is shown (i.e.: "Base Case(s)"), and the actual proof is written in a color that will not be displayed to the students.

In order for TMS to work, the TabletPC needs to have the screen extended to the VGA-out screen rather than having it mirror the tablet's primary screen. The student view of the slides only displays the ink surface, not the toolbar and status bar. Since most projectors do not currently have the ability to rotate the image 90 degrees, I was inspired by the effect of "letter-boxed" movies to create the illusion of a portrait display. Figure 2 shows what is displayed by TMS in landscape-mode via VGA-out.
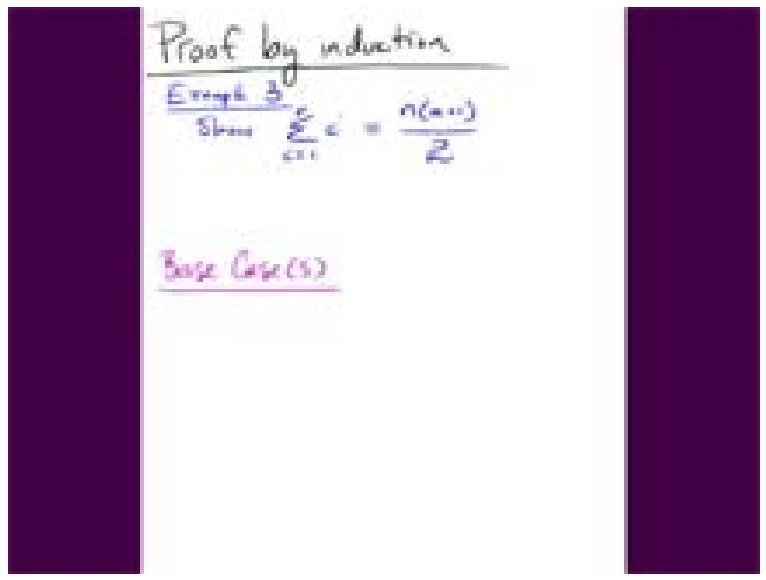

Figure 2: TMS Application student view with example notes for same proof as Figure 1 displayed.

\section{CLASS PREPARATION AND RE-USE OF TMS SLIDES}

A major concern of mine in moving to a new presentation system for my discrete mathematics course was prep time. I had taught the class several times before and had a well-tuned deck of slides. Each of the past two semesters, it was simply a matter of wiping off in-class notes from portions of slides, or rewriting slides if that wasn't realistic, as I reviewed the deck for the coming day's class. As I was teaching the class four days a week during summer session, the time overhead was even more paramount than if I were teaching during the regular semester where class met twice a week.

I had never measured the time it took to prepare existing slides for renewed use each term, so I could only go from the recollection of how long it seemed to take as compared to this term. In previous semesters I took time to review all of the slides and make some modifications. I would also spend time using tissues and water to "delete" material I had written in class the previous semester where possible. If the notes covered too large an area, or were intermixed too closely with the portions I did not want to delete, I would simply rewrite the slide. For each 75-minute lesson, I was able to recreate the slides using TMS in under an hour. I believe this is about the same amount of time I would spend in previous semesters.

The slides were now ready for use in class. Since they were in an electronic form, a copy of the slide deck could be used and marked in class. The originals would still be clean and ready to use in future semesters, requiring minor, if any, time investment above electronically flipping through them before class to refresh my memory.

\section{USE IN CLASS}

In class, I connected the VGA-out of the TabletPC to the cable for the projector and positioned it on a small podium on the desk. The TabletPC has a 12.1" screen and has its resolution set to $1024 \times 768$. From there I use it in much the same way as I use the stage of an overhead projector. One difference from using slide decks is that I did not need to switch between several slides for longer proofs - I could simply continue to scroll. A more significant difference is that if I misjudged the amount of space that a proof step would require, I could simply insert space in the middle of the slide and have the remaining ink moved.

It was also nice to be able to have notes to myself on the slide itself. It felt more natural than having them next to the overhead projector where I would need to glance to the side to check that I was mentioning all of the points I had planned to mention in class. Another benefit was that I did not need to concern myself with where to place slides as I completed using them, or having the slides getting incorrectly ordered due to things such as a student asking to see a previous slide.

At the start of class, I would open the original document and then save it to a directory created for that semester's documents. At the end of class, I would save the marked up version to that directory. If I noticed any errors on the slides, I would then open the original version in the "clean" directory, make the required corrections and save the corrected version back to the directory holding the originals. This allowed me to fine-tune the newly created slides for the next semester while the experience of using them in class was fresh in my mind.

\section{USE DURING OFFICE HOURS}

When students come to ask questions during office hours, lecture notes can play an integral role in the process. There are times when a student thinks they may have simply copied some information incorrectly from the board and would like to compare their notes to those presented. There are also times when a student knows they didn't copy down what you wrote because 
they were paying attention to the discussion and did not want to divide that attention.

If a chalk or white board was the presentation medium, unless you captured the end result using a capture device of some sort $[8,12]$ there may be no way to quickly assist the student towards their goal. If you have detailed notes that you follow, and have them arranged in the same way as you write them on the board, it is possible that you could support this. However, if you varied your presentation that day at all, it could increase the difficulty of this task.

With an electronic presentation system such as TMS, you and the student can look at exactly what was shown in class that day. However, unlike using transparencies, it does not involve the process of reviewing and flipping between multiple transparencies, which I have found to be cumbersome in the past. There is also no concern about keeping the transparencies in proper order. On several occasions students came to office hours with the goal of copying down the details of a proof that they were following in class, but not taking detailed notes on because they didn't want to be distracted from the explanation. On several occasions I was able to work with another student while the first was copying the information they wanted from the slides because they insisted they didn't have questions for me - they just wanted to fill in gaps in their notes for the day. In previous semesters I would insist upon walking through the slides with the student to assure that they were not accidentally rearranged or dropped and lost. This time I was content to be in the vicinity to answer any unexpected questions.

In situations where there is a concept that the student coming to office hours did not follow, and wants to see again in the same manner, just slower, having an exact record of what they just saw in class can be very useful. Even if we think we are presenting something in the same way, it is possible to change variable names or the order of steps that are interchangeable. To us, there is no difference, but to a student it could appear to be very different. Though it can be argued that the goal should be for the student to see that there is no difference, for the student's immediate question, it can be frustrating and potentially confusing. For this purpose the TMS slides were as effective as transparencies.

\section{SHARING NOTES WITH THE TEACHING ASSISTANT}

When working with teaching assistants (TAs) it is very important that you are all following the same, or at least very similar, paths to solutions. If the TA is presenting a different proof style unintentionally (there are times when a different approach is a good idea) a student can become more confused in the process of attempting to integrate the two techniques applied to the same problem. To avoid this, I like to be able to provide my TAs with examples of how I approach proofs in class with the students.

Previously, I had accomplished this by making photocopies of samples of my notes. I did not copy all of my notes due to the time involved in manually copying the transparencies. Since these were grayscale copies, some of the colors I used that looked fine in class would not show up. Others that were different colors in class and clearly showed the stages of the proofs appeared as the same color in the copies and the stage demarcations were lost to the TA when they reviewed the notes.

Using an electronic system, after class I could simply e-mail the TA the set of slides from the class session that just ended. The TA could then easily skim through as many of these as they chose and see all of the details and stages clearly. If a student came in during office hours with a question about a certain problem from class, the TA could easily see exactly what was presented that day. Though not a very scientific measure, it is worth noting that unlike most semesters, over this summer session I did not have any students come saying that the TA did a proof different from how I had done the proof.

\section{OTHER USES}

Besides providing copies of my lecture notes to the TA, I was also able to use TMS to easily supply the TA with detailed outlines for the recitation lessons he presented. I used ink color coding to indicate the problems to present and the solution through which to guide the class. Though I could do this with paper and pens, this had two advantages. First, as with the lecture notes, the TA and I were able to have exact copies of the material. Second, due to the pace of the summer session I often did not create the recitation outlines until the afternoon before the recitation. Since we had a convenient way of electronically exchanging information, we could communicate with more freedom. I was able to e-mail him the slides in a more flexible time frame and he was able to respond with questions before lab in a more flexible time frame.

Another potential use of TMS notes is providing notes to the students. The slide deck can be exported as an HTML document and posted on the class web page. Even if you do not regularly post class notes, you are still able to post or distribute certain notes easily if the need arises. For example, if a student is sick and misses a class, you could opt to send them that day's notes.

Additionally, just as TMS allows for the electronic sharing of your notes with your TA so they can see the way in which you choose to present material and proofs, you can similarly share this information with other faculty members in the department. This can be a quick, effective, and efficient way to facilitate teamteaching or maintain consistency between semesters if desired.

\section{FUTURE PLANS}

My personal experience with the software in my course was a success. I did not feel that I was made to invest more time for class preparations than I had in previous semesters using transparencies. I feel that this time will be greatly reduced the next time I teach the course with the TMS slides. Additionally, when I needed to reorganize existing ink, I did not need to erase the existing ink to redraw it in the new location. In the classroom I felt more satisfied with the fluidity of my presentations and the ability to move within an individual example without needing to flip transparencies.

I was able to enhance my interaction with students during office hours since I could perform activities with students that I had in previous semesters, but without the cumbersome nature of transparencies and without the technology obstructing the process. I was also able to assist more than one student at a time in accomplishing their goals in a way I was unwilling to attempt 
previously. I was able to interact with my teaching assistant and my students in ways that were beneficial to them, which were not readily available in previous semesters.

There are still features that could be enhanced or added to the Tablet Mylar Slides application. Enhancements would be along the lines of more robust HTML export options or the ability to rearrange slides across your individual decks. An example of an addition is more options for hiding parts of the slide. Although TMS allows you to scroll the slide, or write hidden notes to your self, there is currently no way to selectively cover part of a slide as you could with a piece of paper for traditional transparencies. Another example of an addition would be ways in which external objects (e.g.: pre-drawn items, images) could be integrated into the slides.

The current version of the TMS application is being made available to interested faculty with the anticipation that other examples of utility will be compiled. Additionally, as others use the software, other suggestions will undoubtedly arise. A suggestion already offered by a faculty member at a law school who has downloaded the software is the ability to add screen "snippings" to the document as "hidden" notes, similar to the written self-notes currently supported.

\section{ACKNOWLEDGEMETNS}

My thanks to Allison Druin, Ben Bederson, and William Gasarch for inspiration and support, and to Microsoft University Relations for the Toshiba Portege 3500 TabletPC used in the classroom.

\section{REFERENCES}

[1] Abowd, G., "Classroom 2000: An Experiment with the Instrumentation of a Living Educational Experience, " IBM Systems Journal, Special Issue on Pervasive Computing, Volume 38, Number 4, October 1999, pp.508-530.

[2] Anderson, R., Anderson, R., Hoyer, C., Simon, B., Videon, V., and Wolfman, S., "Lecture Presentation for the TabletPC," Workshop on Advance Collaborative Environments, 2003.
[3] BIRD Note-taking System, http://www.cs.umd.edu/ egolub/AVIAN/BIRD.

[4] Cheng, R., "On-line Large Screen Display System for Computer Instruction," Proceedings of the ACM SIGCSESIGCUE Technical Symposium on Computer Science and Education, February 1976, pp.179-181.

[5] Classroom Presenter, http://www.cs.washington.edu/education/dl/presenter.

[6] Good, L., and Bederson, B., "CounterPoint: Creating Jazzy Interactive Presentations," HCIL Tech Report \#2001-03. University of Maryland, College Park, MD 20742, 2001.

[7] Levine, D., "Computer-controlled Display Demonstration of Dynamic Concepts in Computer Science," Proceedings of the ACM SIGCSE-SIGCUE Technical Symposium on Computer Science and Education, February 1976, pp.192-199.

[8] Mimio Whiteboard Capture System, http://www.mimio.com.

[9] Plaisant, C., Rose, A., Rubloff, G., Salter, R., and Shneiderman, B., "The Design of History Mechanisms and Their Use in Collaborative Educational Simulations," Proceedings of the Computer Support for Collaborative Learning 1999 Conference, pp.348-359.

[10] Simon, B., Anderson, R. and Wolfman, S., “Activating Computer Architecture with Classroom Presenter," Workshop on Computer Architecture Education, 2003.

[11] Tablet Mylar Slides, http://www.cs.umd.edu/ egolub/TabletMylarSlides.

[12] WhiteboardPhoto, http://www.websterboards.com/products/wbp.html. 